Received: 25 September 2017

Accepted: 7 February 2018

Published online: 16 February 2018

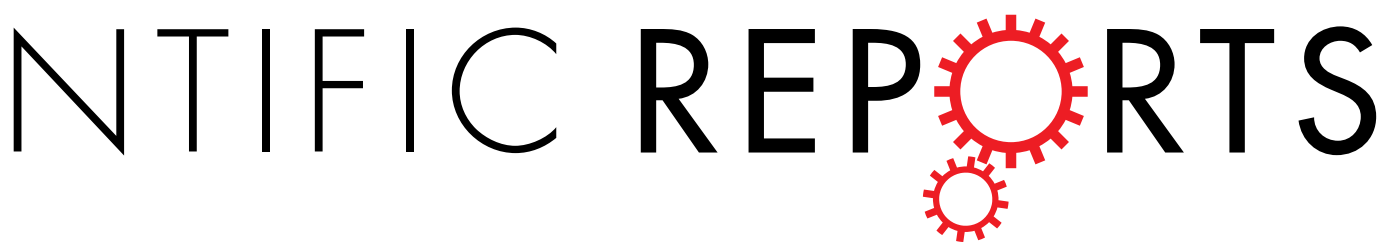

\title{
A Meta-Surface Antenna Array Decoupling (MAAD) Method for Mutual Coupling Reduction in a MIMO Antenna System
}

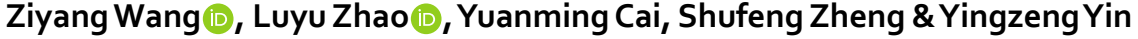

In this paper, a method to reduce the inevitable mutual coupling between antennas in an extremely closely spaced two-element MIMO antenna array is proposed. A suspended meta-surface composed periodic square split ring resonators (SRRs) is placed above the antenna array for decoupling. The metasurface is equivalent to a negative permeability medium, along which wave propagation is rejected. By properly designing the rejection frequency band of the SRR unit, the mutual coupling between the antenna elements in the MIMO antenna system can be significantly reduced. Two prototypes of microstrip antenna arrays at $5.8 \mathrm{GHz}$ band with and without the metasurface have been fabricated and measured. The matching bandwidths of antennas with reflection coefficient smaller than -15 $\mathrm{dB}$ for the arrays without and with the metasurface are $360 \mathrm{MHz}$ and $900 \mathrm{MHz}$ respectively. Using the meta-surface, the isolation between elements is increased from around $8 \mathrm{~dB}$ to more than $27 \mathrm{~dB}$ within the band of interest. Meanwhile, the total efficiency and peak gain of each element, the envelope correlation coefficient (ECC) between the two elements are also improved by considerable amounts. All the results demonstrate that the proposed method is very efficient for enhancing the performance of MIMO antenna arrays.

The surge in video traffic as well as the number of mobile terminal users have boosted the development in wireless communication technologies and systems. As the whole world is moving toward the era of 5-th generation mobile communication, the demand for even higher spectrum and energy efficiency will be everlasting ${ }^{1}$.

In this circumstance, the MIMO/Massive MIMO technology has become an indispensable part of the future wireless communication systems. MIMO technology has the potential to significantly enhance spectrum efficiency by utilizing multiple yet independent data streams existing between the transmitter and the receiver where multiple antennas are installed ${ }^{2}$. While Massive MIMO takes a step further, it uses antenna array with a few hundred elements simultaneously to serve dozens of mobile terminal in limited spectrum and time ${ }^{1}$. In practical applications where space is always limited, antenna arrays, either at the base station end or in a mobile terminal, must be compact in size. Therefore, the inter-element distance is only a fraction of wavelengths, causing inevitable mutual coupling. Mutual coupling will degrade the array performance in the following aspects:

(1) Side-lobes might occur and it will affect the beam-scanning capability of the array ${ }^{3,4}$.

(2) The SNR of the array will be deteriorated as unwanted coupling between elements will cause data streams received by other antenna elements rather than radiating into free space ${ }^{2}$.

(3) The envelop correlations between elements become high thus data throughput is significantly reduced ${ }^{5,6}$.

(4) Gain and total efficiency of the array will drop since effective radiated power are coupled elsewhere and dissipated on the loads of the antenna elements ${ }^{2}$.

Consequently, the mutual coupling reduction technique, or termed as the antenna decoupling technique has drawn great attention from both the academia as well as the industry. The existing and developing decoupling solutions include: neutralization lines ${ }^{7,8}$; decoupling with parasitic scatters ${ }^{9,10}$; eigen-mode decomposition

Xidian University, National Key Laboratory of Antennas and Microwave Technology, Xi'an, 710071, People's Republic of China. Ziyang Wang and Luyu Zhao contributed equally to this work. Correspondence and requests for materials should be addressed to L.Z. (email: lyzhao@xidian.edu.cn) 
(a)

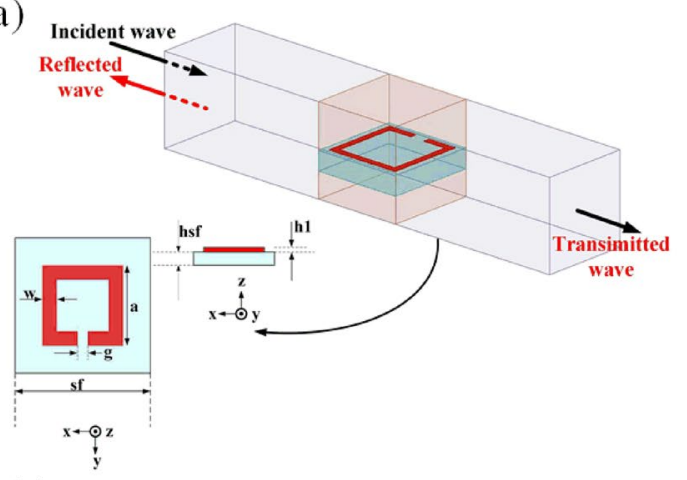

(c)

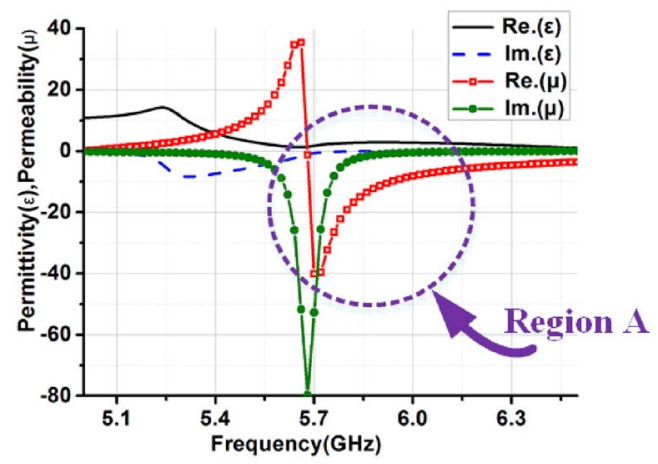

(b)

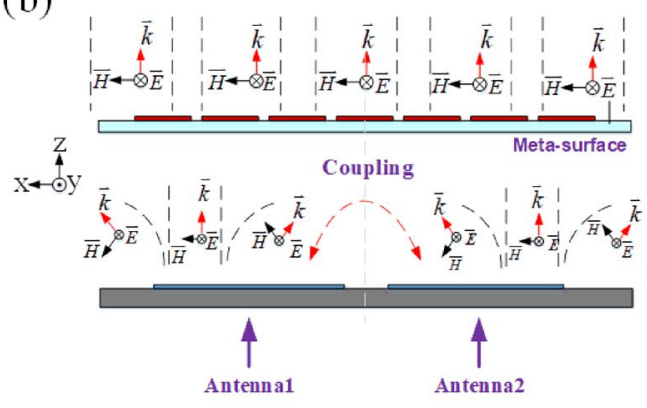

(d)

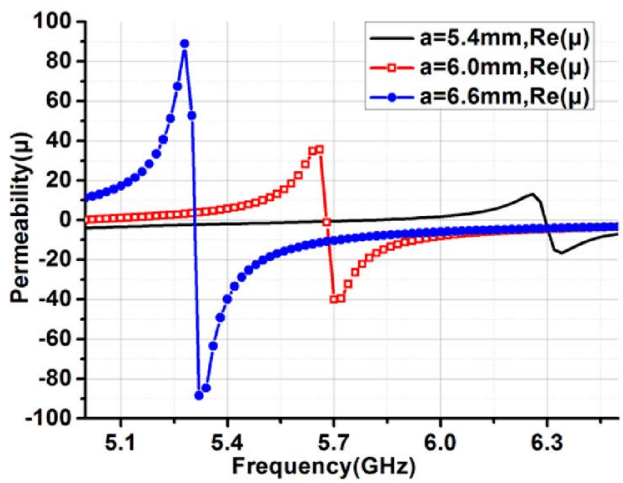

Figure 1. (a) The electromagnetic model to extract design parameters; (b) Radiated field distribution of two coupled antenna elements in an antenna array with the metasurface. (c) The permittivity and permeability of SRR unit; and (d) Simulated permeability of SRR unit with respect to different $a$ values.

techniques ${ }^{11-14}$ and passive decoupling networks ${ }^{15-19}$. However, only a few of them can be readily extended to Massive MIMO antenna arrays with dozens of elements. Metamaterial based or inspired solutions ${ }^{20-25}$ are also becoming popular, but most of them are very effective for arrays with limited number of antennas where inter-element spacing is moderate (no less than $0.1 \lambda_{0}$ ).

In the paper, a metasurface suspended over an antenna array is proposed for antenna decoupling purpose. The idea of covering a metasurface over an antenna for performance enhancement is not new, but most of them are not used for array antenna decoupling, they are used

- To form a Fabry-Perot resonator type antenna for gain enhancement ${ }^{26-28}$.

- For low-profile antenna (array) design ${ }^{29}$.

- To reduce the Radar Cross Section (RCS) of the antenna ${ }^{30}$.

- For beamforming or beam scanning applications $s^{31,32}$.

To the best of the authors' knowledge, it is for the first time that a metasurface covering with negative permeability characteristic is used for antenna decoupling. The proposed MAAD method for compact antenna arrays utilizing meta-surface cover possess the following remarkable features:

(1) Both the decoupling and matching bandwidths of the array with the metasurface are very broad without any extra matching measures;

(2) It can be applied to compact antenna arrays where inter-element spacing is extremely small (smaller than $\left.0.02 \lambda_{0}\right)$

(3) Because of the periodic nature of metasurfaces, it has great potential to be applied to Massive MIMO antenna arrays where a few hundreds of antennas are excited simultaneously;

(4) It is also able to enhance gain and efficiency of the antenna array thanks to the focusing capability of the metasurface.

\section{Design Method}

Antenna Array Decoupling Mechanism in a Negative Permeability Medium. As shown in Fig. 1 (a) and (b), a suspended meta-surface with SRRs as its unit cell over a two-element antenna array is used to illustrate the working mechanism of the MAAD method. An isotropic homogeneous slab model (Fig. 1a) is used to calculate the effective permittivity, the effective permeability of the metasurface unit using the extraction methods well-known ${ }^{33-36}$. The resonant frequency and the negative permeability frequency region of the SRRs 


\begin{tabular}{|l|l|l|l|}
\hline Parameters & Values & Parameters & Values \\
\hline$a$ & 6.0 & $w$ & 0.5 \\
\hline$g$ & 1.2 & $s f$ & 7 \\
\hline$h_{1}$ & 0.035 & $h_{2}$ & 1.5 \\
\hline
\end{tabular}

Table 1. Design Parameters of the SRR unit (Unit: $\mathrm{mm}$ ).

are determined by the physical dimension of the ring and the size of the gap as shown in Table 1 . The equivalent values of permittivity and permeability for the proposed SRRs unit are shown in Fig. 1(c). As shown in Fig. 1(c), in $5.8 \mathrm{GHz}$ frequency band, the real part of permittivity is positive, while the real part of permeability is negative. It is obvious that tuning the parameter $a$, the negative permeability frequency region of the SRRs can easily be manipulated as shown in Fig. 1(d).

To reveal the mutual coupling reduction mechanism using the MAAD method, let's consider a two-element MIMO antenna array shown in Fig. 1(b). When antenna 1 is excited, electromagnetic waves propagate in the way shown in Fig. 1(b), stray coupling component $A_{0} e^{j k x}$ which travels along the minus X-direction will cause induced current on antenna 2 thereby create mutual coupling between the two antennas.

A piece of suspended metasurface over the antenna array is able to create a region with negative permeability yet positive permittivity $\left(\mu_{r}<0, \epsilon_{r}>0\right)$, as shown in Fig. 1(c). In this region, the wavenumber can be expressed as:

$$
k=k_{0} \cdot \sqrt{-\left|\mu_{r}\right| \cdot\left|\epsilon_{r}\right|}=j k_{0} \cdot \sqrt{\left|\mu_{r}\right| \cdot\left|\epsilon_{r}\right|}
$$

which is purely imaginary. (Only positive solution of equation (1) is valid).

In this case, the corresponding $\mathrm{x}$-component of the electric field travelling along the $-\mathrm{x}$ direction, $A_{0} \mathrm{e}^{\mathrm{jkx}}$ can be further expressed as:

$$
A_{0} e^{j k x} \cdot e^{j \omega t}=A_{0} e^{j\left(j k_{0} \cdot \sqrt{\left|\mu_{r}\right| \cdot\left|\epsilon_{r}\right|}\right) x} \cdot e^{j \omega t}=A_{0} e^{-k_{0} \sqrt{\left|\mu_{r}\right| \cdot\left|\epsilon_{r}\right|} x} \cdot e^{j \omega t}
$$

Equation (2) shows that electromagnetic wave travelling along minus X-direction of the metasurface is evanescent. In this manner, the wave creating mutual coupling between the two antennas is rejected. When the wave radiated by antennas propagate along Z-direction, while the magnetic field component is in the X-direction, radiation is assured by the anisotropic nature of the metasurface.

Design of two-element Microstrip Antenna Array with a Metasurface. To further demonstrate the effectiveness of the MAAD method for mutual coupling suppression, a two-element microstrip antenna array (Array1) as shown in Fig. 2(a) is used in this paper for illustration purpose. The physical dimensions of the array are listed in Table 2. The edge-to-edge distance between the elements $a d=1 \mathrm{~mm}$, which is approximately $0.02 \lambda_{0}$ at $5.8 \mathrm{GHz}$. The patches are printed on a FR4 substrate with the dielectric constant of 4.4 and the loss tangent of 0.02 . The simulated S-parameters of the Arrayl are plotted in Fig. 2(d). It is obvious from Fig. 2(d) that although the antenna elements are well matched at the $5.8 \mathrm{GHz}$ band, but the isolation between the two antenna elements is no more than $8 \mathrm{~dB}$, which is very poor. Effective decoupling measures must be taken.

A metasurface consists of periodic of SRRs is introduced to improve the inter-element isolation of the array in Fig. 2(b). The SRRs are printed on a substrate with the relative dielectric constant of 2.65 and loss tangent of 0.001. Four dielectric posts are introduced to provide mechanical support for the metasurface and the antenna array (Array2). The dimensions in Fig. 2(b) are also shown in Table 2.

Simulated S-parameters of the Array2 with the metasurface are superposed in Fig. 2(d). It can be seen from Fig. 2(d) that isolation performance is significantly enhanced by introducing the metasurface covering while matching performance is not ideal, this is understandable since the antennas are no longer matched to a free-space medium. Then two U-shape slots which is commonly used to enhance microstrip antenna matching ${ }^{37}$ are etched on the antenna near the feeding probe (Array3) as shown in Fig. 2(c). The matching bandwidth of Array3 with $\left|\mathrm{S}_{11}\right|$ smaller than $-15 \mathrm{~dB}$ is about $900 \mathrm{MHz}$, which is about 2.5 times of the Array1 without metasurface. While the isolation between the elements of Array3 is all below $-27 \mathrm{~dB}$ from $5.49 \mathrm{GHz}$ to $6.0 \mathrm{GHz}$, which is increased by a large amount compared to Arrayl. Moreover, the profile of the Array 3 with the metasurface is only increased by about $0.09 \lambda_{0}$, which is acceptable.

Field Distribution. To further reveal the underlying working mechanisms of the array with the metasurface, the vector magnetic field distributions on a cutting surface within XOZ-plane are investigated using ANSYS HFSS. As shown in Fig. 3(a) and (b). Without the metasurface, the vector H-field is rotating in Region A with the metasurface, the $\mathrm{H}$-field in Region $\mathrm{B}$ is almost along the $\mathrm{X}$-axis. On the same cutting plane, the Poynting vector distributions are also plotted and shown in Fig. 3(c) and (d) for the arrays without and with metasurface, respectively. The Poynting vectors for the array with metasurface are almost in parallel to the Z-axis without any lateral radiation.

The contours of the electric field for the arrays without and with metasurface are also shown in Fig. 3(e) and (f). In the simulation, Ant 1 is excited while Ant 2 is terminated with matched load. It can be concluded from Fig. 3(e) and (f) that, in the array without metasurface, stray coupling from antenna 1 will be coupled to antenna 
(a)

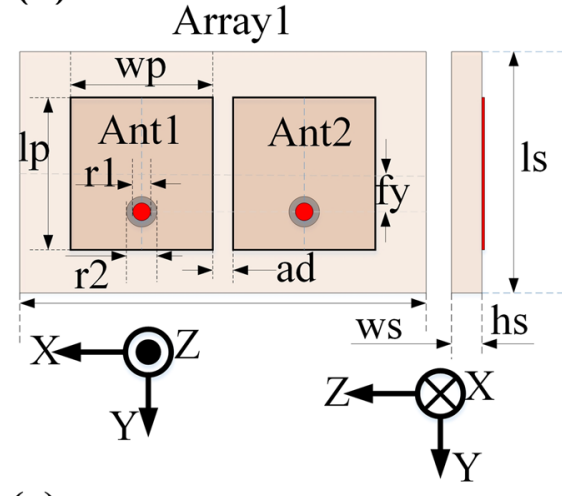

(c)

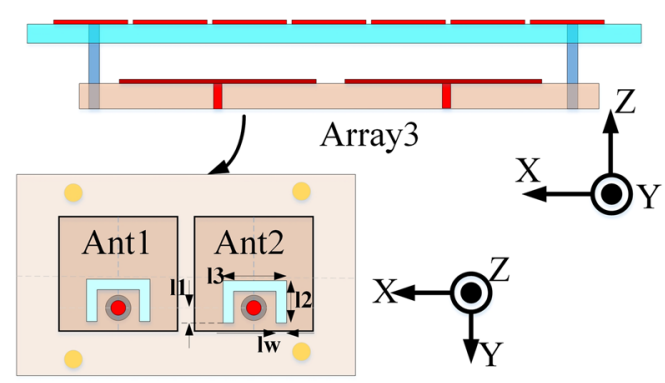

(b)

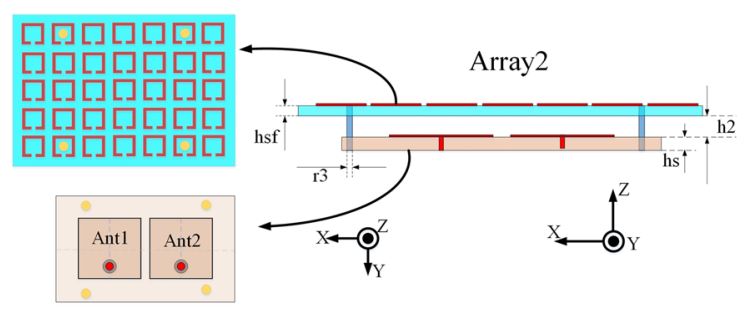

(d)

Figure 2. (a) Top view (left) and side view (right) of the two-element patch Array1; (b) Top view (left) and side view (right) of the two-element patch Array2 with a metasurface; (c) Top view (left) and side view (right) of the improved two-element patch Array3 with a metasurface; (d) Simulated S-parameters of the Array1, Array2 and Array3.

\begin{tabular}{|l|l|l|l|l|l|}
\hline Parameters & Values & Parameters & Values & Parameters & Values \\
\hline$w s$ & 40 & $h 2$ & 4.8 & $h s f$ & 1.5 \\
\hline$l s$ & 26 & $r 1$ & 0.65 & $l w$ & 0.6 \\
\hline$h s$ & 3 & $r 2$ & 1.5 & $l 1$ & 3.0 \\
\hline$w p$ & 12.4 & $a$ & 6 & $l 2$ & 4.2 \\
\hline$l p$ & 12.5 & $w$ & 0.5 & $l 3$ & 4 \\
\hline$a d$ & 1 & $g$ & 1.2 & - & - \\
\hline$f y$ & 3.3 & $s f$ & 7 & - & - \\
\hline
\end{tabular}

Table 2. Design Parameters of the SRR unit (Unit: $\mathrm{mm}$ ).

2 and absorbed by the load. While with the help of the metasurface, the wave front concentrate toward the normal direction of the microstrip antenna.

Parametric Studies. Parametric studies are conducted to analyze the sensitivity of different design parameters. Two clusters S-parameter curves are obtained with respect to different lengths of the SRR, $a$; and different heights of the metasurface covering, $h 2$. They are superposed in Fig. 4(a) and (b), respectively. It can be seen that parameter $a$, the length of the SRR is more sensitive in the design, since the length of the SRR will directly affect the negative permeability frequency band of the SRR, as shown in Fig. 1(d). Therefore, the band-rejection frequency of the metasurface will shift to lower frequencies if parameter $a$ increases, and it will shift to higher frequencies if parameter $a$ decreases, which is consistent with the observation in Fig. 1(d).

The isolation performance of the antenna array with the metasurface is not very height-sensitive as shown in Fig. 4(b). This is reasonable since the rejected propagation band of the metasurface remains unchanged, yet the return losses of the array vary significantly because of different parasitic effect of the metasurface with respect to different meta-surface height.

In order to explore the optimum configuration of the SRR units on the metasurface, the matching and isolation performances for the array with metasurface of $6 \times 4,8 \times 6$ and $7 \times 5$ periodic SRR units are also compared in Fig. 4(c). Form Fig. 4(c), we can conclude that the both the matching and isolation performance of the array with only $6 \times 4$ SRR units are not ideal. The isolation performance for the array with $8 \times 6$ SRR units is better than the array with $7 \times 5 \mathrm{SRR}$ units only within the bandwidth from about $5.65 \mathrm{GHz}$ to no more than $5.8 \mathrm{GHz}$. If we look 

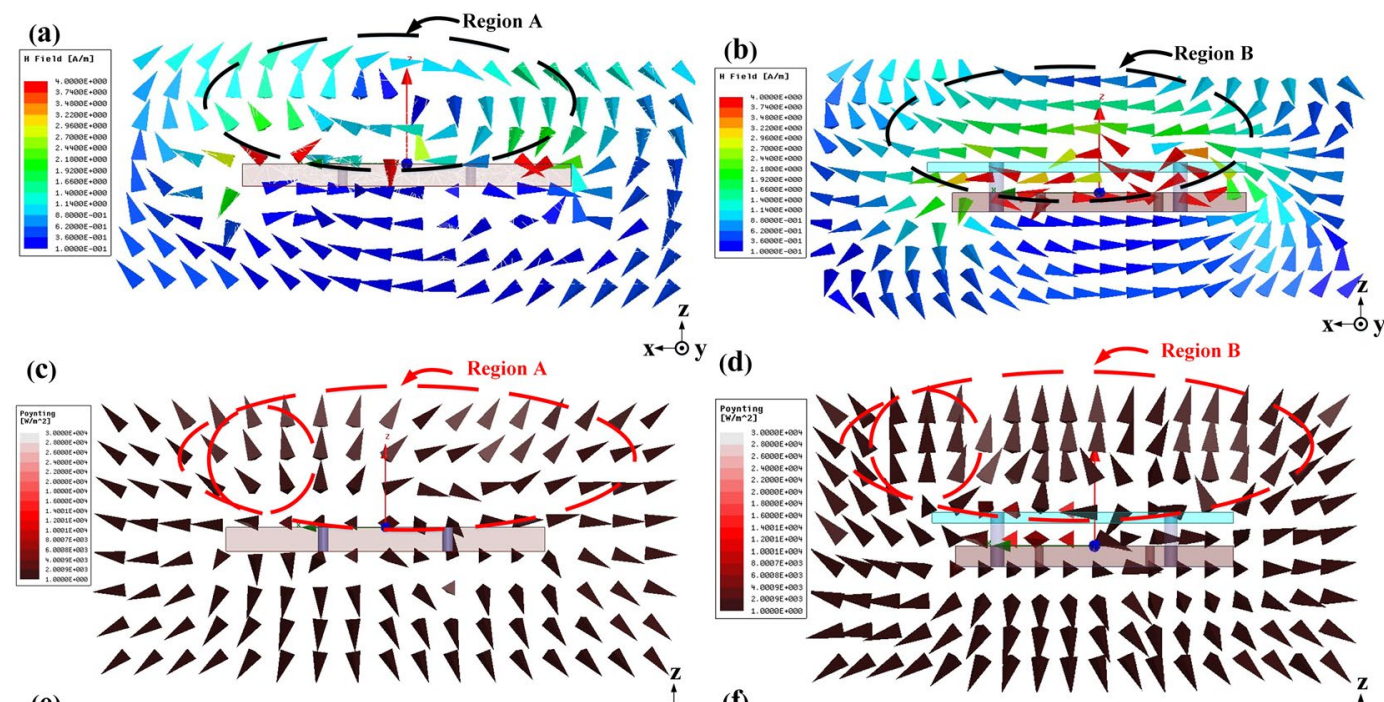

(e)
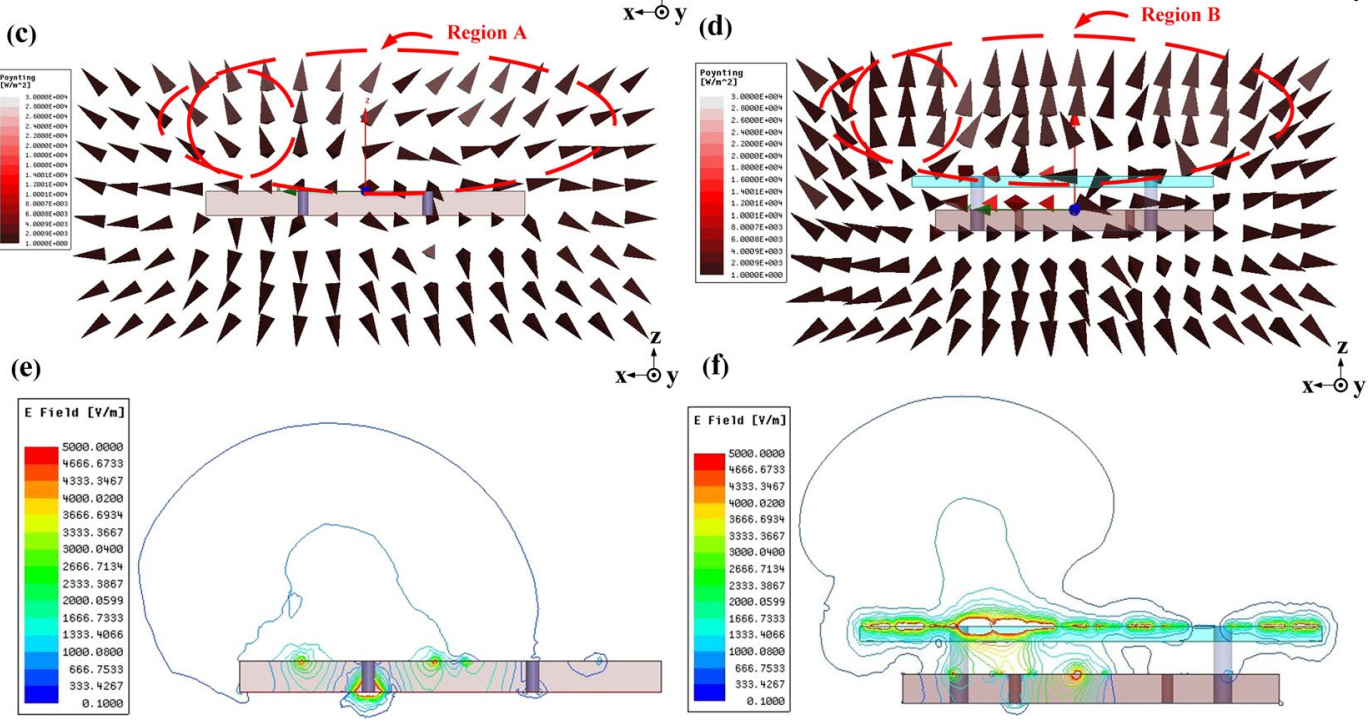

Figure 3. (a) Simulated vector magnetic field distribution on a cutting surface in the XOZ-plane for array without metasurface; (b) Simulated vector magnetic field distribution on a cutting surface in the XOZ-plane for array with metasurface; (c) Simulated Poynting vector distribution on a cutting surface in the XOZ-plane for array without metasurface; (d) Simulated Poynting vector distribution on a cutting surface in the XOZ-plane for array with metasurface; (e) Simulated electric field contours on a cutting surface in the XOZ-plane when Ant 1 is excited while Ant 2 terminated with matched load for array without metasurface; (f) Simulated electric field contours on a cutting surface in the XOZ-plane when Ant 1 is excited while Ant 2 terminated with matched load for array with metasurface. All of the plots are at $5.8 \mathrm{GHz}$.

at the band from $5.6 \mathrm{GHz}$ to $6 \mathrm{GHz}$, the isolation performance of the array with $7 \times 5 \mathrm{SRR}$ units is actually better than the array with $8 \times 6$ SRR units.

Furthermore, to quantify the effectiveness of the decoupling method with the metasurface, antenna array without any metasurfaces but with different edge-to-edge spacing values, $a d$ are simulated. The results are superposed with the isolation for Array3 in Fig. 4(d). Within the frequency band of interest, the isolation of the array with the metasurface is equivalent to the array without the metasurface but whose inter-element spacing is about 35 times larger.

\section{Results}

Scattering Parameters. Both Array1 and Array3 are fabricated and measured. The scattering parameters are measured using Keysight E5080A vector network analyzer and they are superposed in Fig. 5, which is consistent with simulation results shown in Fig. 2(d). The measured highest isolation of Array3 can reach to more than $40 \mathrm{~dB}$ level. The measured Array3's operating bandwidth with $\left|S_{11}\right|<-15 \mathrm{~dB}$ and $\left|\mathrm{S}_{21}\right|<-25 \mathrm{~dB}$ is about $400 \mathrm{MHz}$.

Radiation Parameters. To further confirm the performance of the array with the metasurface, the fabricated samples of the arrays are put into the SATIMO SG-24 near field scanner (shown in Fig. 6(f)) to evaluate the radiation related characteristics, including: total efficiencies, gains, and envelope correlation coefficients. Since the mutual coupling is reduced by more than $20 \mathrm{~dB}$ and more powers are directed to the normal direction of the antennas, both the total efficiencies and the peak gains of the array with metasurface will be enhanced. As shown in Fig. 6(c), the total efficiency for the array with the metasurface (Array3) is 20\% higher as compared to the array without any metasurfaces (Array1). The radiation patterns of the arrays are also shown in Fig. 6(a) and (b) while the peak gains with respect to different frequencies are shown in Fig. 6(d), showing around 2 dB improvement.

The envelop correlation coefficient (ECC) is an important figure of merit for any MIMO enabled antenna systems. It can be calculated from measured complex field patterns by using 
(a)

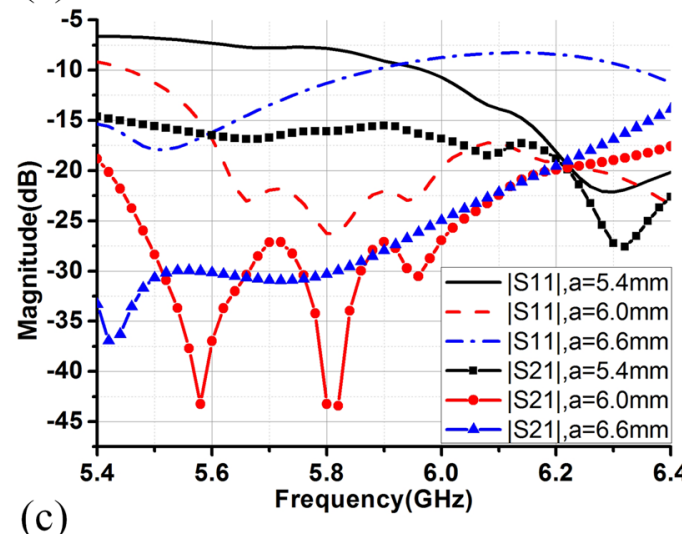

(c)

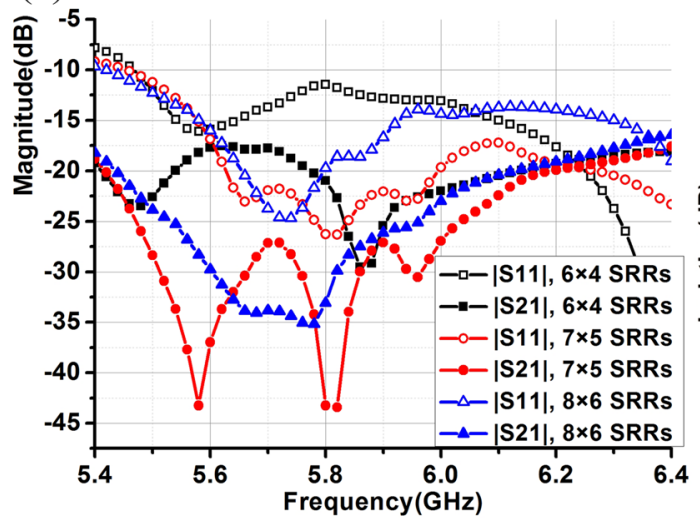

(b)

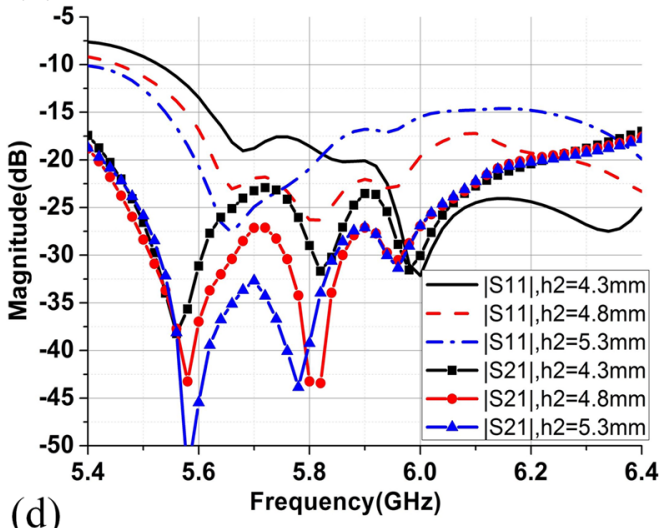

(d)

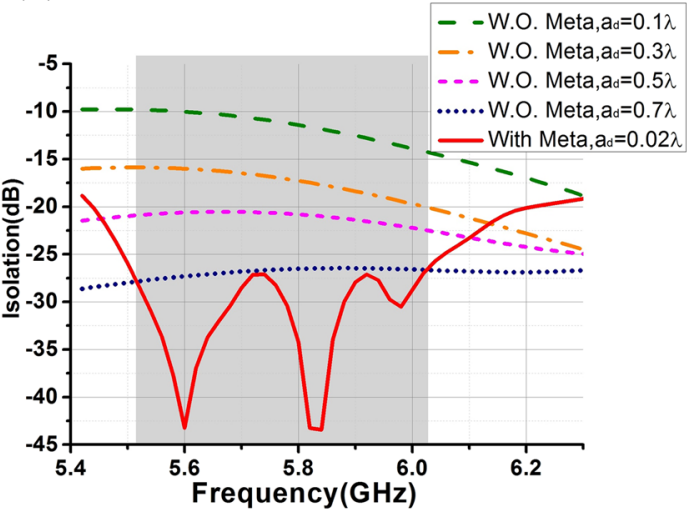

Figure 4. (a) Simulated S-parameters of the array with the metasurface with respect to different a- the length of the SRR, values; (b) Simulated S-parameters of the array with the metasurface with respect to different h2- the height of the metasurface, values; (c) Simulated S-parameters of the antenna array with the metasurface with respect to different number of SRR units; (d) Simulated isolations for arrays with different ad values and the isolation for the array with a metasurface.

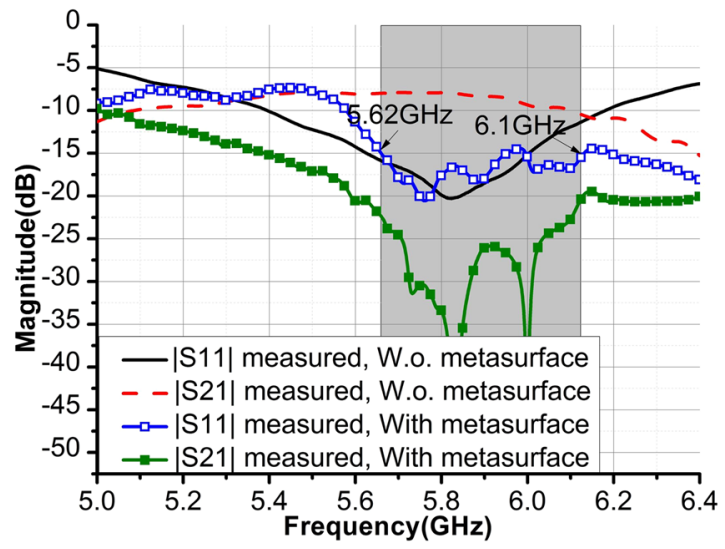

Figure 5. Measured S-parameters of the arrays with and without metasurface.

$$
\begin{gathered}
\rho_{e}=\frac{\left|\iint_{4 \pi}\left[\vec{E}_{1}(\theta, \phi) \cdot \vec{E}_{2}(\theta, \phi)\right] d \Omega\right|^{2}}{\iint_{4 \pi}\left|\vec{E}_{1}(\theta, \phi)\right|^{2} d \Omega \cdot \iint_{4 \pi}\left|\vec{E}_{1}(\theta, \phi)\right|^{2} d \Omega} \\
\vec{E}_{1}(\theta, \phi) \cdot \vec{E}_{2}(\theta, \phi)=\vec{E}_{\theta 1}(\theta, \phi) \cdot \vec{E}_{\theta 2}^{*}(\theta, \phi)+\vec{E}_{\phi 1}(\theta, \phi) \cdot \vec{E}_{\phi 2}^{*}(\theta, \phi)
\end{gathered}
$$


(a)

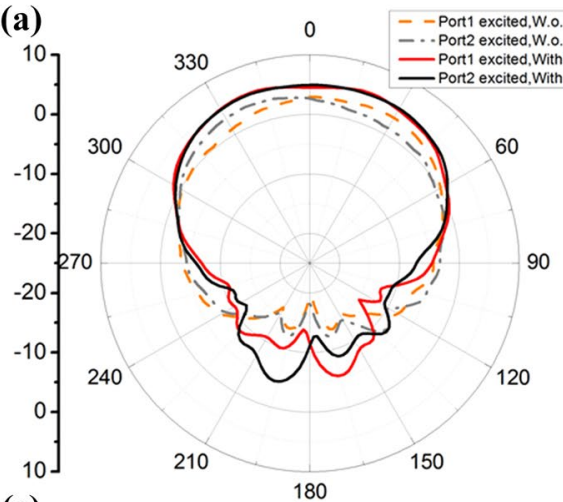

(c)

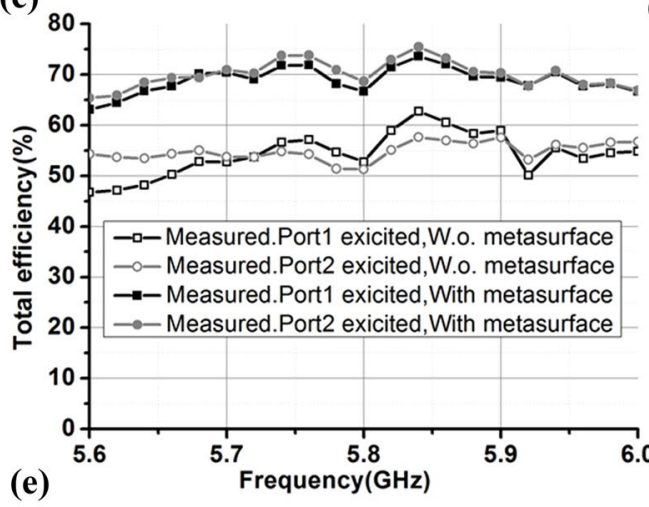

(e)

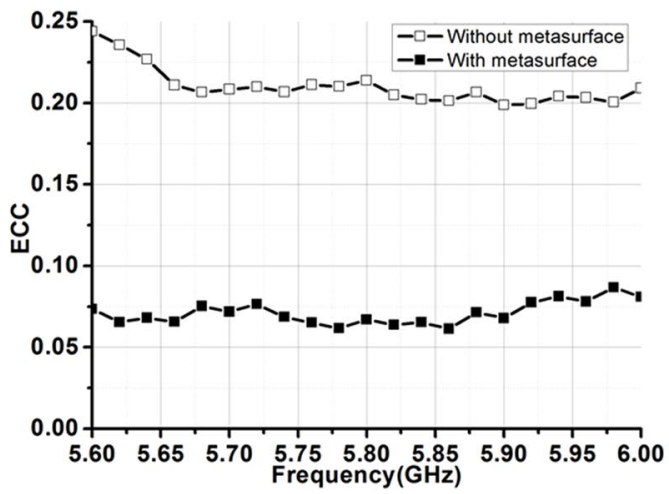

(b)

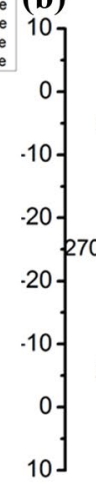

(d)

- - Port1 excited,W.O. metesurface

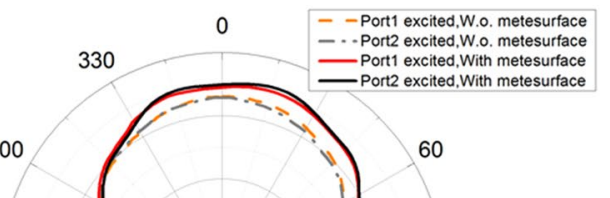

240

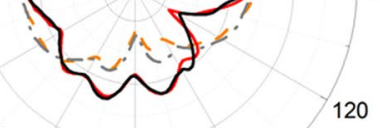

210

120
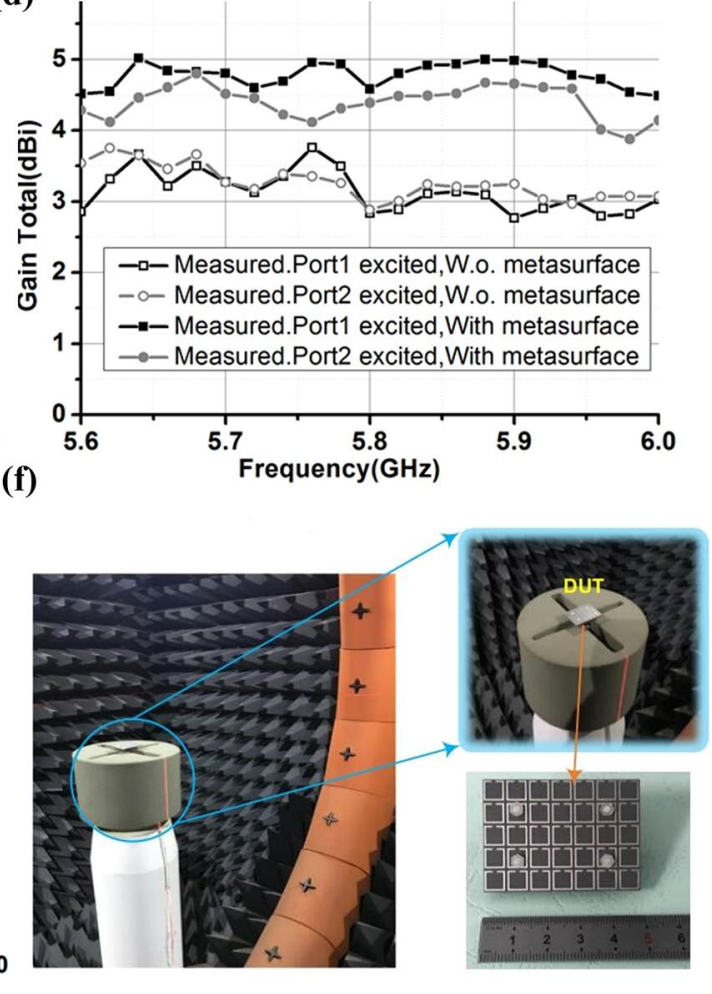

Figure 6. (a) Measured radiation patterns for the arrays at $5.8 \mathrm{GHz}$ with and without metasurface in the $\mathrm{XOZ}$ plane; (b) Measured radiation patterns for the arrays at $5.8 \mathrm{GHz}$ with and without metasurface in the $\mathrm{YOZ}$ plane; (c) Measured total efficiencies of the arrays with and without metasurface; (d) Measured peak gains of the arrays with and without metasurface on different frequencies; (e) Measured and calculated ECCs for the arrays with and without metasurface. (f) Radiation measurement setup and DUT configuration.

where $\vec{E}_{1}(\theta, \phi)$ is the measured electric filed vector radiated by antenna 1 while the another antenna port is terminated by a $50 \Omega$ matched load ${ }^{38}$. The calculated ECCs for the arrays with and without metasurface are given in Fig. 6(e). It is clear that by introducing the metasurface, the ECC has improved from 0.25 to less than 0.08 , which will definitely lead to larger channel capacity and diversity gain.

\section{Conclusion}

A brand-new decoupling method making use of metasurface covering with negative permeability is introduced for the first time in this paper. The metasurface has the capability of reject unwanted radiation as well as reducing mutual coupling in an antenna array without affecting other performances.

The design can be conformal and low-profile, and most importantly, it can be applied to arrays with extremely small element spacing while most existing decoupling solutions cannot. Unlike other decoupling method, as isolation after decoupling becomes higher, the matching bandwidth is not reduced at all, on the contrary, it is even broader.

Thanks to the periodic nature of the metasurface with SRRs, the proposed MAAD method has great potential to be applied to antenna arrays with dozens of elements, which are commonly utilized in Massive MIMO arrays 
and phased arrays. Using the metasurface decoupling method proposed in this paper to decouple more than 64 element antenna array is undergoing and results will be reported on in due time.

\section{Methods}

Numerical calculation of the decoupling antenna array was performed using Finite Element method, which is conducted by commercial software, ANSYS HFSS. The microstrip antennas are printed in a commercial FR4 printed circuit board with the relative permittivity 4.4 and loss tangent 0.02 . Moreover, the substrate printed with square split ring resonators was a commercial printed circuit board (F4B) with the relative permittivity 2.65 and loss tangent 0.001 . In experiments, we used the Agilent vector network analyzer to measure the transmission and reflection coefficients between antennas. The far-field radiation patterns are evaluated using a microwave near-field chamber.

\section{References}

1. Larsson, E. G., Edfors, O., Tufvesson, F. \& Marzetta, T. L. Massive MIMO for next generation wireless systems. IEEE Communications Magazine 52(2), 186-195 (2014).

2. Jensen, M. A. \& Wallace, J. W. A review of antennas and propagation for MIMO wireless communications. IEEE Transactions on Antennas and Propagation 52(11), 2810-2824 (2004).

3. Steyskal, H. \& Herd, J. S. Mutual coupling compensation in small array antennas. IEEE Transactions on Antennas and Propagation 38(12), 1971-1975 (1990).

4. Pozar, D. M. Considerations for millimeter wave printed antennas. IEEE Transactions on Antennas and Propagation 31(5), 740-747 (1983).

5. Lu, L., Li, G. Y., Swindlehurst, A. L., Ashikhmin, A. \& Zhang, R. An Overview of Massive MIMO: Benefits and Challenges. IEEE Journal of Selected Topics in Signal Processing 8(5), 742-758 (2014).

6. Taluja, P. S. \& Hughes, B. L. Diversity Limits of Compact Broadband Multi-Antenna Systems. IEEE Journal on Selected Areas in Communications 31(2), 326-337 (2013).

7. Wang, Y. \& Du, Z. A Wideband Printed Dual-Antenna With Three Neutralization Lines for Mobile Terminals. IEEE Transactions on Antennas and Propagation 62(3), 1495-1500 (2014).

8. Su, S. W., Lee, C. T. \& Chang, F. S. Printed MIMO-antenna system using neutralization-line technique for wireless USB-dongle applications. IEEE Transactions on Antennas and Propagation 60(2), 456-463 (2012).

9. Lau, B. K. \& Andersen, J. B. Simple and efficient decoupling of compact arrays with parasitic scatterers. IEEE Transactions on Antennas and Propagation 60(2), 464-472 (2012).

10. Zhao, L. \& Wu, K. L. A Decoupling Technique for Four-Element Symmetric Arrays With Reactively Loaded Dummy Elements. IEEE Transactions on Antennas and Propagation, vol. 62, no. 8, pp. 4416-4421 (2014).

11. Coetzee, J. C. \& Yu, Y. Port decoupling for small arrays by means of an eigenmode feed network. IEEE Transactions on Antennas and Propagation 56(6), 1587-1593 (2008).

12. Volmer, C., Weber, J., Stephan, R., Blau, K. \& Hein, M. A. An eigen-analysis of compact antenna arrays and its application to port decoupling. IEEE Transactions on Antennas and Propagation 56(2), 360-370 (2008).

13. Yeung, L. K. \& Wang, Y. E. Mode-based beamforming arrays for miniaturized platforms. IEEE Trans. Microw. Theory Tech. 57(1), $45-52(2009)$

14. Wang, K., Li, L. \& Eibert, T. F. Comparison of Compact Monopole Antenna Arrays With Eigenmode Excitation and Multiport Conjugate Matching. IEEE Transactions on Antennas and Propagation 61(8), 4054-4062 (2013).

15. Andersen, J. \& Rasmussen, H. Decoupling and descattering networks for antennas. IEEE Transactions on Antennas and Propagation 24(6), 841-846 (1976).

16. Chen, S. C., Wang, Y. S. \& Chung, S. J. A decoupling technique for increasing the port isolation between strongly coupled antennas. IEEE Transactions on Antennas and Propagation 56(12), 3650-3658 (2008).

17. Zhao, L., Yeung, L. K. \& Wu, K. L. A coupled resonator decoupling network for two-element compact antenna arrays in mobile terminals. IEEE Transactions on Antennas and Propagation 62(5), 2767-2776 (2014).

18. Qian, K., Zhao, L. \& Wu, K. L. An LTCC Coupled Resonator Decoupling Network for Two Antennas. IEEE Transactions on Antennas and Propagation 63(10), 3199-3207 (2015).

19. Amjadi, S. M. \& Sarabandi, K. Mutual Coupling Mitigation in Broadband Multiple-Antenna Communication Systems Using Feedforward Technique. IEEE Transactions on Antennas and Propagation 64(5), 1642-1652 (2016).

20. Yang, F. \& Rahmat-Samii, Y. Microstrip antennas integrated with electromagnetic band-gap EBG structures: A low mutual coupling design for array applications. IEEE Transactions on Antennas and Propagation 51(10), 2936-2946 (2003).

21. Wei, K., Li, J. Y., Wang, L., Xing, Z. J. \& Xu, R. Mutual Coupling Reduction by Novel Fractal Defected Ground Structure Bandgap Filter. IEEE Transactions on Antennas and Propagation 64(10), 4328-4335 (2016).

22. Ghosh, S., Tran, T. N. \& Le-Ngoc, T. Dual-Layer EBG-Based Miniaturized Multi-Element Antenna for MIMO Systems. IEEE Transactions on Antennas and Propagation 62 (8), 3985-3997 (2014).

23. Mavridou, M., Feresidis, A. P. \& Gardner, P. Tunable Double-Layer EBG Structures and Application to Antenna Isolation. IEEE Transactions on Antennas and Propagation 64(1), 70-79 (2016).

24. Pan, B. C. et al. Reduction of the spatially mutual coupling between dual-polarized patch antennas using coupled metamaterial slabs. Scientific Reports 6, 30288 (2016).

25. Al-Hasan, M. J., Denidni, T. A. \& Sebak, A. R. Millimeter-Wave Compact EBG Structure for Mutual Coupling Reduction Applications. IEEE Transactions on Antennas and Propagation 63(2), 823-828 (2015).

26. Feresidis, A. P., Goussetis, G., Wang, S. \& Vardaxoglou, J. C. Artificial magnetic conductor surfaces and their application to lowprofile high-gain planar antennas. IEEE Transactions on Antennas and Propagation 53(1), 209-215 (2005).

27. Wang, N. et al. Wideband Fabry-Perot Resonator Antenna With Two Complementary FSS Layers. IEEE Transactions on Antennas and Propagation 62(5), 2463-2471 (2014).

28. Qin, F. et al. A Simple Low-Cost Shared-Aperture Dual-Band Dual-Polarized High-Gain Antenna for Synthetic Aperture Radars. IEEE Transactions on Antennas and Propagation 64(7), 2914-2922 (2016).

29. Malekpoor, H. \& Jam, S. Improved Radiation Performance of Low Profile Printed Slot Antenna Using Wideband Planar AMC Surface. IEEE Transactions on Antennas and Propagation 64(11), 4626-4638 (2016).

30. Liu, Y. et al. Wideband RCS Reduction of a Slot Array Antenna Using Polarization Conversion Metasurfaces. IEEE Transactions on Antennas and Propagation 64(1), 326-331 (2016).

31. Jiang, M., Chen, Z. N., Zhang, Y., Hong, W. \& Xuan, X. Metamaterial-Based Thin Planar Lens Antenna for Spatial Beamforming and Multibeam Massive MIMO. IEEE Transactions on Antennas and Propagation 65(2), 464-472 (2017).

32. Cameron, T. R. \& Eleftheriades, G. V. Analysis and Characterization of a Wide-Angle Impedance Matching Metasurface for Dipole Phased Arrays. IEEE Transactions on Antennas and Propagation 63(9), 3928-3938 (2015).

33. Smith, D. R., Schultz, S., Markos, P. \& Soukoulis, C. M. Determination of effective permittivity and permeability of metamaterials from reflection and transmission coefficients. Phys. Rev. B 65, 195104 (2002). 
34. Ziolkowski, R. W. Design, fabrication, and testing of double negative metamaterials. IEEE Transactions on Antennas and Propagation 51(7), 1516-1529 (2003).

35. Chen, X., Grzegorczyk, T. M., Wu, B. I., Pacheco, J. Jr. \& Kong, J. A. Robust method to retrieve the constitutive effective parameters of metamaterial. Phys. Rev. E 70, 016608 (2004).

36. Smith, D. R., Vier, D. C., Koschny, T. \& Soukoulis, C. M. Electromagnetic parameter retrieval from inhomogeneous metamaterials. Phys. Rev. E 71, 036617 (2005)

37. Tong, K. F., Luk, K. M., Lee, K. F. \& Lee, R. Q. A broad-band U-slot rectangular patch antenna on a microwave substrate. IEEE Transactions on Antennas and Propagation 48(6), 954-960 (2000).

38. Vaughan, R. G. \& Andersen, J. B. Antenna diversity in mobile communications. IEEE Trans. Veh. Technol. 36(4), 147-172 (1987).

\section{Acknowledgements}

This work is sponsored by National Natural Science Foundation of China (Grant No. 61701366).

\section{Author Contributions}

L.Y.Z. conceived the basic concept. Z.Y.W., S.F.Z. and Y.M.C. did theoretical analysis. Z.Y.W. and L.Y.Z. conducted simulations fabrication and measurements. Z.Y.W. and L.Y.Z. wrote the manuscript based on input from all authors. All authors contributed to the discussion.

\section{Additional Information}

Competing Interests: The authors declare no competing interests.

Publisher's note: Springer Nature remains neutral with regard to jurisdictional claims in published maps and institutional affiliations.

(c) (i) Open Access This article is licensed under a Creative Commons Attribution 4.0 International License, which permits use, sharing, adaptation, distribution and reproduction in any medium or format, as long as you give appropriate credit to the original author(s) and the source, provide a link to the Creative Commons license, and indicate if changes were made. The images or other third party material in this article are included in the article's Creative Commons license, unless indicated otherwise in a credit line to the material. If material is not included in the article's Creative Commons license and your intended use is not permitted by statutory regulation or exceeds the permitted use, you will need to obtain permission directly from the copyright holder. To view a copy of this license, visit http://creativecommons.org/licenses/by/4.0/.

(c) The Author(s) 2018 